\title{
Análisis de los datos maternos y perinatales en pacientes con hipospadias en un periodo de 25 años
}

\author{
Sánchez Zalabardo JM*, Bono Ariño A**, Gracia Romero J***, López López JA*. \\ *Servicio de Urología. Hospital Clínico Universitario "Lozano Blesa". Zaragoza. **Servicio de Urología. Hospital \\ "San Jorge". Huesca. ***Servicio de Cirugía Pediátrica. Hospital Universitario "Miguel Servet". Zaragoza.
}

Actas Urol Esp. 2007;31(7):776-780

\section{RESUMEN}

\section{ANÁLISIS DE LOS DATOS MATERNOS Y PERINATALES EN PACIENTES CON HIPOSPADIAS EN UN PERIODO DE 25 AÑOS}

Introducción: El objetivo del estudio es definir los factores de riesgo materno-fetales que pueden influir en la aparición de hipospadias al nacimiento y en la gravedad del mismo.

Material y Métodos: Se han recogido los datos de 614 recién nacidos con hipospadias operados en nuestra comunidad en los últimos 25 años (1972-1998). Para su estudio se han dividido en tres grupos de gravedad de la malformación (proximales, medios y distales) y en dos periodos de tiempo, antes y después de 1980, analizándolos en función de los posibles factores predisponentes.

Resultados: El 9\% presentaban hipospadias proximales, un 13,6\% medios y un 77,5\% distales. Se ha encontrado una relación estadísticamente significativa entre la gravedad del hipospadias y el bajo peso al nacimiento, retraso en el crecimiento intrauterino, prematuridad y existencia de malformaciones asociadas $(\mathrm{p}<0,001)$. No se ha encontrado significación estadística entre la gravedad del hipospadias y la edad materna, hábitos tóxicos, existencia de enfermedades o exposición a radiaciones en el embarazo. A partir del año 1980, los pacientes presentan hipospadias más severos y una edad gestacional menor.

Conclusiones: Existen ciertos acontecimientos en el embarazo que pueden favorecer el desarrollo de hipospadias en el feto. Se esta produciendo un incremento en el número de pacientes con hipospadias y gravedad de estos desde 1980.

Palabras clave: Hipospadias. Epidemiología. Factores predisponentes.

\section{ABSTRACT}

\section{MATERNAL AND PERINATAL DATA OF HYPOSPADIC IN A 25 YEAR PERIOD}

Objective: To analyze maternal and perinatal risk factors related to the onset and severity of hypospadias.

Material and Methods: Data of 614 boys operated on hypospadias in our county during 25 years (1972-1998) has been studied. The patients were divided into 3 groups according the malformation severity: proximal, middle, and distal hypospadias. We use two periods, before and after 1980 to analyze the differences in risk factors along the time.

Results: There were 9\% of proximal hypospadias, 13,6\% middle hypospadias, and 77,5\% distal hypospadias. We found statistical significance in several risk factors related to severe hypospadias: low birth weight, intrauterine growth restriction, preterm births, and associated malformations $(p<0,001)$. We did not find statistical significance with maternal age, toxic exposure, associated diseases, and x-ray exposure during pregnancy. After 1980, there were more severe hypospadias and less gestational age.

Conclusions: Several events during pregnancy can contribute to the development of hypospadias in the fetus. In our experience, since 1980 there is a rise in the number and severity of hypospadias. 
$\mathrm{E}$ 1 objetivo de este estudio es identificar factores de riesgo maternos o fetales en nuestra serie de pacientes hipospádicos que puedan predisponer a la aparición de hipospadias más severos. La incidencia de hipospadias ha sido estimada en 3,2 por cada 1.000 varones nacidos vivos ${ }^{1-3}$. Esta afección es más frecuente en la raza blanca que en la negra y también se ha señalado mayor frecuencia en italianos y judíos ${ }^{4}$. La mayoría de los autores admiten que el hipospadias es la consecuencia de una etiología multifactorial. Una demanda mayor de gonadotropina coriónica humana placentaria durante el primer trimestre del embarazo ${ }^{5}$ podría ser la razón de la mayor incidencia encontrada en gemelos homocigóticos, 8,5 veces mayor. Sólo en un $30 \%$ de los hipospadias severos se consigue identificar la etiologia ${ }^{6}$, teniendo un papel importante los tratamientos hormonales durante el embarazo. Silver ${ }^{7}$ justifica la estimulación estrogénica en la fertilización in vitro como responsable del aumento en la incidencia de hipospadias en niños concebidos mediante esta técnica. Dentro de los factores ambientales, $\mathrm{Safe}^{8}$ habla de la exposición a estrógenos como causa de malformaciones y disminución de las cifras del espermiograma. $\mathrm{Chia}^{9}$ defiende que el aumento en la incidencia de cáncer testicular, hipospadias e infertilidad se debe a agentes químicos utilizados por el hombre que actúan alterando la función endocrina, provocando un desarrollo anómalo en el eje reproductivo. En estudios experimentales con ratas, se ha demostrado que la administración de distintos tipos de pesticidas provocaba grados variables de malformaciones congénitas, en relación con el grado de afinidad de las distintas moléculas de los pesticidas con el receptor de andrógenos ${ }^{10,11}$. Irgens $^{12}$ analizando alrededor de un millón de nacimientos en el registro noruego de nacimientos desde 1970 a 1993, encuentra un aumento de incidencia de hipospadias en padres que trabajan como mecánicos de automóviles. En España, García y cols. ${ }^{13}$ desarrollaron un estudio de casos y controles en la Comunidad Valenciana para valorar la relación entre la exposición a pesticidas en los agricultores y la prevalencia de malformaciones congénitas, encontrando un aumento en la "odds ratio" en madres que realizaron tareas agrícolas entre el mes anterior a la con- cepción y el primer trimestre de embarazo. La dieta vegetariana materna presenta un riesgo mas elevado de hipospadias en el nacimiento ${ }^{14}$ por un posible efecto de los fitoestrógenos durante el desarrollo embrionario. La edad materna tiene también un papel importante en la aparición de defectos esofágicos, malformaciones cardiacas e hipospadias ${ }^{15-17}$.

\section{MATERIAL Y MÉTODOS}

Hemos realizado un estudio retrospectivo, de cohortes históricas, mediante revisión de las historias de 614 pacientes intervenidos de hipospadias, 518 en el Servicio de Cirugía Pediátrica del Hospital Universitario Miguel Servet de Zaragoza, desde la apertura del mismo en el año 1972 hasta 1998 y 76 en el Servicio de Urología del Hospital Clínico Universitario Lozano Blesa de Zaragoza desde 1978 hasta 1997. Con los datos obtenidos, hemos efectuado un estudio estadístico básico e inferencial. Para la comparación entre variables cuantitativas con una distribución normal hemos usado el análisis de la varianza (ANOVA) y en aquellas con distribución no normal la prueba de Kruskall-Wallis. Las comparaciones dos a dos se han establecido mediante la prueba de NeumanKeuls. Respecto a las variables cualitativas, las comparaciones se han hecho mediante el test de chi cuadrado. Se ha dividido a los pacientes en varios grupos, según el tipo de hipospadias, proximal, medio y distal y el año de nacimiento. El año 1980 coincide con la mediana de distribución de los pacientes según su año de nacimiento, por lo que lo hemos escogido como punto de corte de comparación.

\section{RESULTADOS}

De los 614 pacientes estudiados, 560 presentan datos válidos para ser incluidos en el estudio. 50 pacientes $(9 \%)$ presentaban hipospadias proximales, 76 pacientes $(13,6 \%)$ hipospadias medios, y $434(77,5 \%)$ hipospadias distales. La edad media de realización de la uretroplastia fue de 5 años.

Los datos del recién nacido y del embarazo junto con su significación estadística se resumen en la Tabla 1.

En 151 casos $(20,7 \%)$ existen malformaciones asociadas al hipospadias, siendo hasta un $61,3 \%$ 
Tabla 1. Datos según el tipo de hipospadias y nivel de significación estadística.

\begin{tabular}{lcccc}
\hline Tipo de hipospadias & Distales & Medios & Proximales & p \\
\hline N (\%) & $434(77,5 \%)$ & $76(13,6 \%)$ & $50(8,9 \%)$ & \\
Edad materna (años) & 28,4 & 28,8 & 28,9 & \\
Edad paterna (años) & 31,6 & 31,5 & 32,02 & \\
Edad cirugía (años) & 5 & 5,8 & 3,8 & \\
Peso al nacimiento (g) & 3204 & 2919 & 2564 & $<0,001$ \\
Bajo peso (<2500 g) (\%) & $31(12,8 \%)$ & $11(19 \%)$ & $18(52,9 \%)$ & $<0,001$ \\
Edad gestacional (sem) & 39,11 & 38,4 & 37,4 & $<0,001$ \\
Pretérmino (<37 sem) & $22(5 \%)$ & $6(14,6 \%)$ & $10(30,3 \%)$ & $<0,001$ \\
Exposición a tóxicos & 4 & 0 & 0 & n.s \\
Exposición a RX & $11(3,5 \%)$ & $3(4,7 \%)$ & $5(11,36 \%)$ & 0,05 \\
Problemas de fertilidad & $4(1,2 \%)$ & $2(3,1 \%)$ & $5(11,36 \%)$ & 0,05 \\
Malformaciones asociadas & $65(20 \%)$ & $24(31,5 \%)$ & $27(61,3 \%)$ & $<0,001$ \\
\hline
\end{tabular}

en los hipospadias proximales. Las malformaciones más frecuentemente asociadas son las urológicas $(13,8 \%)$ seguidas por las malformaciones cardiacas, craneofaciales, y síndromes polimalformativos. Hasta un 50\% de los recién nacidos con menos de $2.500 \mathrm{~g}$ presentaban malformaciones asociadas, con una relación estadísticamente significativa $(\mathrm{p}<0,001)$.

En cuatro casos existía un embarazo gemelar, presentando los dos hermanos hipospadias en un caso, asociados a nacimiento pretérmino (33 semanas) y bajo peso al nacimiento (1.700 y $1.800 \mathrm{mg})$. Los dos hermanos presentaban síndromes polimalformativos.

Cuarenta mujeres presentaron problemas durante el embarazo, siendo los más frecuentes la amenaza de aborto, diabetes, hipertensión arterial e infecciones del tracto urinario. Un 5\% de las madres (27) consumieron tabaco durante el embarazo.

Hemos encontrado diferencias estadísticamente significativas al comparar, según la gravedad del hipospadias, la existencia o no de problemas de fertilidad previos, exposiciones a radiaciones ionizantes, y enfermedades durante el embarazo. No hemos encontrado diferencias en relación con la edad materna, el tabaquismo, los viajes durante el embarazo, la actividad profesional materna o la exposición a tóxicos durante el embarazo.
No existen diferencias entre los datos maternos al dividir los pacientes en dos grupos, antes y después de 1980 (Tabla 2). En los últimos 20 años se ha duplicado el número de recién nacidos con menos de $2.500 \mathrm{~g}$, y la edad gestacional media ha descendido de 39,4 a 38,5 semanas, con diferencias estadísticamente significativas entre los dos grupos $(\mathrm{p}$ 0,002 y $\mathrm{p}<0,001$ respectivamente).

No hemos hallado diferencias estadísticamente significativas entre las edades paterna y materna ni el tipo de hipospadias en los dos periodos de tiempo estudiados.

\section{DISCUSIÓN}

El no haber encontrado diferencias en las edad media paterna y materna de los niños con hipospadias antes y después de 1980 parece contradecir la evidente tendencia actual de aumento en la edad de las madres a la hora de tener su primer hijo. En el estudio realizado por Fisch ${ }^{17}$ la incidencia de grados de hipospadias más severos aumenta en las madres de 35 años hasta en un $20 \%$ comparada con la incidencia en las madres de 20 años. Otros autores como Kallen ${ }^{18}$ y Porter ${ }^{19}$ también encuentran diferencias en la incidencia de hipospadias conforme aumenta la edad materna, citando la edad de menor riesgo en las primíparas de 20 años. Sin embargo, en un estudio reciente, Bianca ${ }^{20}$ establece los grupos de edad "extremos", mujeres menores de 20 y mayores de 40 años, como los de mayor riesgo de presentar hipospadias, por alteración en las concentraciones de hormonas sexuales durante el embarazo en ambos grupos de edad.

Compartimos la teoría de que junto con datos ya conocidos de factores genéticos y hereditarios que únicamente explican un pequeño porcentaje de hipospadias, existe una serie de factores ambientales que sobre un feto predispuesto provocarían la aparición del hipospadias. A pesar de 
Tabla 2. Datos según la fecha de nacimiento y nivel de significación estadística.

\begin{tabular}{lccc}
\hline & Antes de $\mathbf{1 9 8 0}$ & Después de $\mathbf{1 9 8 0}$ & $\mathbf{p}$ \\
\hline Hipospadias distales & 213 & 192 & n.s. \\
Hipospadias medios & 22 & 41 & n.s. \\
Hipospadias proximales & 11 & 31 & $<0,001$ \\
Edad media materna (años) & 27 & 28 & n.s. \\
Edad media paterna (años) & 30 & 31 & n.s. \\
Peso medio al nacimiento (g) & 3249 & 3020 & 0,001 \\
Bajo peso (<2500 g) (\%) & $20(11 \%)$ & $48(23,3 \%)$ & 0,002 \\
Edad media gestacional (sem) & 39,4 & 38,5 & $<0,001$ \\
Malformaciones asociadas & $72(28,5 \%)$ & $62(23,2 \%)$ & 0,19 \\
\hline
\end{tabular}

la posible influencia de la dieta vegetariana en la aparición del hipospadias ${ }^{14}$ no nos consta ningún caso en nuestra serie.

Aunque no exista una relación directa con el tabaco $^{21}$, los hábitos tóxicos maternos, sobre todo el tabaco están directamente relacionados con la aparición de bajo peso al nacimiento, que está íntimamente relacionado con la aparición de grados más severos de hipospadias y malformaciones asociadas ${ }^{22}$. En nuestra serie, no hemos encontrado relación estadísticamente significativa entre la ingesta de fármacos, los hábitos tóxicos o exposición a tóxicos en el embarazo y el tipo de hipospadias del niño, aunque en los hipospadias escrotales sean más frecuentes. Este dato está en discordancia con los estudios que relacionan la actividad profesional materna con la aparición de hipospadias en el recién nacido ${ }^{23-26}$.

Hay relación estadísticamente significativa entre la existencia o no de problemas de fertilidad en los padres y el tipo de hipospadias del niño $(\mathrm{p}<0,001)$. Los hijos de padres sometidos a tratamientos de fertilidad presentan en nuestro estudio hipospadias más severos. Este dato hablaría a favor del papel que desempeñan los factores hormonales en la producción del hipospadias, y confirma los hallazgos de Silver ${ }^{7}$.

El haber tenido o no un embarazo anormal tiene una relación estadísticamente significativa con el tipo de hipospadias del niño $(\mathrm{p}<0,05)$. Además de la relación con la toma de alcohol durante el embarazo, Carbone ${ }^{23}$ también considera la presencia de enfermedades crónicas en la madre como factor de riesgo independiente en la aparición de malformaciones genitourinarias.

No hemos encontrado relación entre los viajes durante el embarazo, haber trabajado, haber tenido enfermedades durante el embarazo o haber tomado anticonceptivos antes del embarazo con el tipo de hipospadias del niño.

Después del año 1980 hay mas niños con bajo peso al nacimiento, con una diferencia estadísticamente significativa $(\mathrm{p}<0,002)$. Este dato no es exclusivo de los pacientes con hipospadias, sino que la disminución del peso medio del recién nacido es generalizada al conjunto de la población, probablemente por el cambio en el estilo de vida y hábitos maternos, aumento de las técnicas de reproducción asistida, con partos múltiples y embarazos de riesgo.

Como ya es sabido ${ }^{27}$, y así lo confirman nuestros resultados, existe mayor incidencia de malformaciones congénitas asociadas al hipospadias en los recién nacidos con bajo peso. Estos pacientes también presentan grado más severos de hipospadias. Para Calderón ${ }^{22}$ la presencia de bajo peso al nacimiento es el factor de riesgo más importante para la aparición de anomalías como el hipospadias y la criptorquidia.

La relación entre la edad gestacional con el tipo de hipospadias del niño es estadísticamente significativa $(\mathrm{P}<0,001)$. Estas diferencias se hacen más claras entre los hipospadias distales y proximales. Hemos encontrado diferencias estadísticamente significativas en las medias de edad gestacional antes y después del año 1980 $(\mathrm{P}<0,001)$. Después de 1980, la edad gestacional es menor y los hipospadias son más severos.

Respecto a las malformaciones asociadas nuestros resultados coinciden con los encontrados en la literatura ya que encontramos malformaciones asociadas en un $25 \%$ de $\operatorname{los} \operatorname{casos}^{28}$ siendo las malformaciones urológicas (14\%) el doble de frecuentes que las cardiacas que son las que le siguen en frecuencia. La relación entre la presencia de malformaciones asociadas con el tipo de hipospadias del niño es estadísticamente significativa. 


\section{CONCLUSIONES}

La existencia de problemas de fertilidad previos y exposiciones a radiaciones ionizantes durante el embarazo pueden favorecer un desarrollo incompleto de la uretra. Los factores de riesgo del recién nacido de presentar hipospadias son el retraso en el crecimiento intrauterino, la prematuridad, y el bajo peso al nacimiento. Se ha producido un incremento en el número de pacientes con hipospadias y de la gravedad de éstos desde 1980 .

\section{REFERENCIAS}

1. Sweet RA, Schrott HG, Kurland R. Study of the incidence of hypospadias in Rochester, Minnesota, 1940-1970, and a case-control comparison of possible etiologic factors. Mayo Clin Proc. 1974 Jan;49(1):52-58.

2. Sorensen HR. Hypospadias with special reference to aetiology. Copenhagen, Ejnar Munksgaard, 1953.

3. Avellan L. The incidence of hipospadias in Sweden. Scand J Plast Reconstr Surg. 1975;9(2):129-139.

4. Welch KJ. Hypospadias. In: Ravitch MM, Welch KJ, Benson CD, et al, editors. Pediatric Surgery. 3rd ed. Chicago: Year Book Medical Publishers 1979;1353-1376.

5. Roberts CJ, Lloyd S. Observations on the epidemiology of simple hypospadias. Br Med J. 1973 Mar 31;1(5856):768770.

6. Boehmer AL, Nijman RJ, Lammers BA, de Coninck SJ, Van Hemel JO, Themmen AP, et al. Etiological studies of severe or familiar hipospadias. J Urol. 2001 Apr;165(4):12461254.

7. Silver-RI, Rodriguez R, Chang TS, Gearhart JP. In vitro fertilization is associated with an increased risk of hypospadias. J Urol 1999;161(6):1954-1957.

8. Safe SH. Endocrine disruptors and human health. Is there a problem?. An update. Environ Health Perspect 2000; 108(6):487-493.

9. Chia SE. Endocrine disruptors and male reproductive function. A short review. Int J Androl. 2000;23 Suppl 2:4546

10. Gray LE Jr, Wolf C, Lambright C, Mann P, Price M, Cooper RL, Ostby J. Administration of potentially antiandrogenic pesticides (procymidone, linuron, iprodione, chlozolinate, p,p' DDE, and ketoconazole) and toxic substances (dibutyl and diethylhexyl phthalate, PCB 169, and ethane dimethane sulphonate) during sexual differentiation produces diverse profiles of reproductive malformations in the male rat. Toxicol Ind Health 1999; 15(1-2):94-118.

11. Mylchreest E, Wallace DG, Cattley RC, Foster PM. Dose dependent alterations in androgen regulated male reproductive development in rats exposed to Di(n butyl) phthalate during late gestation. Toxicol Sci. 2000 May;55(1):143151 .

12. Irgens A, Kruger K, Skorve AH, Irgens LM. Birth defects and paternal occupational exposure. Hypotheses tested in a record linkage based dataset. Acta Obstet Gynecol Scand. 2000 Jun;79(6):465-470.

13. Garcia AM, Fletcher T, Benavides FG, Orts E. Parental agricultural work and selected congenital malformations. Am J Epidemiol. 1999 Jan 1;149(1):64-74.
14. North K, Golding J A. Maternal vegetarian diet in pregnancy is associated with hypospadias. The ALSPAC Study Team. Avon Longitudinal Study of Pregnancy and Childhood. BJU Int. 2000 Jan;85(1):107-113.

15. Hay S, Barbano $\mathrm{H}$. Independent effects of maternal age and birth order on the incidence of selected congenital malformations. Teratology. 1972 Dec;6(3):271-279.

16. Kallen B, Bertollini R, Castilla E, Czeizel A, Knudsen LB Martinez-Frias ML, et al. A joint international study of the epidemiology of hypospadias. Acta Paediatr Scand Suppl. 1986;324:1-52

17. Fisch H, Golden RJ, Libersen GL, Hyun GS, Madsen P, New MI, et al. Maternal age as a risk factor for hypospadias. J Urol. 2001 Mar;165(3):934-936.

18. Kallen B,Bertollini R, Castilla E, Czeizel A, Knudsen LB, Martínez-Frías ML, Mastroiacovo P, Mutchinick O. A joint international study of the epidemiology of hypospadias Acta Paediatr Scand, suppl. 1986;324:1-52.

19. Porter MP, Faizan MK, Grady RW, Mueller BA. Hypospadias in Washington State: maternal risk factors and prevalence trends. Pediatrics. 2005 Apr;115(4):e495499. Epub 2005 Mar 1.

20. Bianca S, Ingegnosi C, Ettore G. Maternal age and hypospadias. Acta Obstet Gynecol Scand. 2005 Apr;84(4):410.

21. Carmicahel SL, Shaw GM, Laurent C, Lammer EJ, Olney RS. Hypospadias and maternal exposure to cigarette smoke. Paediatr Perinat Epidemiol. 2005 Nov;19(6):406412.

22. Calderon JS, Zarante I. Congenital urological anomalies: epidemiological description and associated risk factors in Colombia 2001-2004. Arch Esp Urol 2006;59(1):7-14.

23. Carbone P, Giordano F, Nori F, Mantovani A, Taruscio D, Lauria L, et al. Cryptorchidism and hypospadias in the Sicilian district of Ragusa and the use of pesticides. Reprod Toxicol. 2006 Jul;22(1):8-12. Epub 2006 Mar 10.

24. Vaktskjold A, Talykova LV, Chashchin VP, Nieboer E, Thomassen Y, Odland JO. Genital malformations in newborns of female nickel refinery workers. Scand J Work Environ Health. 2006 Feb;32(1):41-50.

25. Irgens A, Kruger K, Skorve AH, Irgens LM Birth defects and paternal occupational exposure. Hypotheses tested in a record linkage based dataset. Acta Obstet Gynecol Scand 2000;79(6):465-470.

26. Garcia AM, Fletcher T, Benavides FG, Orts E. Parental agricultural work and selected congenital malformations. Am J Epidemiol 1999 Jan 1; 149(1):64-74

27. Kallen B, Bertollini R, Castilla E, Czeizel A, Knudsen LB, Martinez-Frias ML, et al. A joint international study of the epidemiology of hypospadias. Acta Paediatr Scand, suppl 1986;324: 1-52.

28. Wu WH, Chuang JH, Y Ting YC, Lee SY, Hsieh CS. Developmental Anomalies and Disabilities Associated With Hypospadias. J Urol. 2002 Jul;168(1):229-232.

Correspondencia autor: Dr. D. J.M. Sánchez Zalabardo

Servicio de Urología.

Hospital Clínico Universitario "Lozano Blesa"

Avda. San Juan Bosco, 15 - 50009 Zaragoza

Tel.: 976765700

E-mail autor: drsanchezurologo@yahoo.es

Información artículo: Original - Urología pediátrica

Trabajo recibido: diciembre 2006

Trabajo aceptado: marzo 2007 